\title{
ESTRATÉGIAS E METODOLOGIAS DE AJUSTE DE MODELOS HIPSOMÉTRICOS EM PLANTIOS DE Eucalyptus sp.
}

\author{
Andressa Ribeiro ${ }^{1}$, Antonio Carlos Ferraz Filho², José Márcio de Melloº , Maria Zélia Ferreira ${ }^{4}$, \\ Priscila Maria Martins Lisboa ${ }^{5}$, José Roberto Soares Scolforo ${ }^{6}$
}

(recebido: 7 de maio de 2009; aceito: 30 de outubro de 2009)

\begin{abstract}
RESUMO: Conduziu-se este trabalho com o objetivo de testar diferentes estratégias e metodologias de coleta de dados, a fim de realizar ajustes de modelos hipsométricos tradicionais e genéricos para melhor estimar a altura de árvores em plantios de Eucalyptus sp. Foram coletados pares de altura e diâmetro de árvores distribuídas em 36 parcelas, provenientes de plantios pertencentes à empresa Veracel Celulose S.A. Essas foram divididas em diferentes tratamentos conforme idade, região, e combinação de região e idade do plantio, totalizando 14 tratamentos. As metodologias de coleta de dados consistiram na utilização de diferentes números de árvores com medição de altura para formação da base de dados para realização dos ajustes. Foi selecionando como melhor estratégia de ajuste, o modelo de Curtis por parcela e dois modelos genéricos para as diferentes metodologias adotadas, com exceção da metodologia baseada em adição de árvores representativas em todos os quartis diamétricos, que selecionou apenas a estratégia de Curtis por parcela.
\end{abstract}

Palavras-chave: Relação hipsométrica, inventário florestal, modelos tradicionais, modelos genéricos.

\section{STRATEGIES AND METHODOLOGYS FOR ADJUSTMENT OF HYPSOMETRIC MODELS OF Eucalyptus sp. STANDS}

\begin{abstract}
This study aimed at testing different data gathering and adjustment strategies of traditional and generic hypsometric models in order to improve Eucalyptus sp. tree height estimates. Tree height and diameter pairs were colleted in 36 plots, belonging to Veracel Celulose S.A. The data were divided into different treatments according to age, site, and combination of age and site of the stands, totaling 14 treatments. The data gathering strategies consisted in using a different number of trees with height measurement to form the database used for the model adjustments. When considering the data gathering strategies where the first five trees per plot and the first five trees per plot plus the dominants were selected, it was concluded that the best strategies consisted in using the Curtis model adjusted per plot, and the two generic models from Campos \& Leite (2006) and Scolforo (2005). When considering the data gathering strategy in which trees were added based on the absence of an individual on a determined diameter quartiles, only the Curtis model per plot was selected.
\end{abstract}

Key-words: Height-diameter relationship, forest inventory, traditional and generic models.

\section{INTRODUÇÃO}

O setor florestal brasileiro se encontra em plena expansão, apresentando grande competitividade no mercado mundial de produtos florestais. Assim, estudos sobre mensuração florestal, visando à redução de custo é de extremo valor. A medição da altura em povoamentos florestais é de grande importância para estimativa do volume e qualificação da produtividade em determinado local, sendo o volume fundamental na fase de planejamento da produção para qualquer empresa florestal. Das características mensuradas no inventário florestal, a medição da altura é seguramente a mais onerosa. A quantificação do volume de madeira é feita por meio de procedimentos de inventários florestais (SILVA et al., 2007), e nestes aplicam-se relações hipsométricas nas árvores

\footnotetext{
${ }^{1}$ Engenheira Florestal, Mestranda em Engenharia Florestal - Departamento de Ciências Florestais - Universidade Federal do Paraná Av. Pref. Lothário Meissner, 900 - 80210-170 - Jardim Botânico - Curitiba, PR - andressa_ufla@yahoo.com.br

${ }^{2}$ Engenheiro Florestal, Doutorando em Ciências Florestais - Departamento de Ciências Florestais - Universidade Federal de Lavras - Cx. P. 3037 - 37200-000 - Lavras, MG - acferrazfilho@gmail.com

${ }^{3}$ Engenheiro Florestal, Professor Doutor do Departamento de Ciências Florestais - Universidade Federal de Lavras - Cx.P. 3037 37200-000 - Lavras, MG - jmello@dcf.ufla.br

${ }^{4}$ Engenheira Florestal, Mestra em Ciências Florestais - Veracel Celulose S.A - Cx. P. 23 - $45820-970$ - Eunápolis, BA maria.zelia@veracel.com.br

${ }^{5}$ Engenheira Florestal, Mestranda em Meio ambiente e Recursos hídricos - Universidade Federal de Itajubá - Cx. P. 50 - $37500-903$ Itajubá, MG - priscilamlisboa@yahoo.com.br

${ }^{6}$ Engenheiro Florestal, Professor Doutor do Departamento de Ciências Florestais - Universidade Federal de Lavras - Cx. P. 3037 37200-000 - Lavras, MG - jscolforo@dcf.ufla.br
}

Cerne, Lavras, v. 16, n. 1, p. 22-31, jan./mar. 2010 
medidas na parcela, a fim de se estimar a variável altura nas demais árvores que não foram mensuradas, reduzindo custo no processo de medição.

$\mathrm{Na}$ prática, no inventário florestal, mede-se o diâmetro de todas as árvores na parcela e a altura de parte delas e, por meio dos pares altura-diâmetro mensurados, estabelece-se uma equação matemática que possibilite estimar as alturas das demais árvores da parcela. Essas equações matemáticas são conhecidas como relações hipsométricas, as quais buscam estimar as alturas das árvores por meio da relação do diâmetro da árvore a 1,30m do solo e da altura total (THIERSCH, 2007). Essa técnica de mensuração, já descrita por Ker \& Smith (1957), resulta em uma sensível redução nos custos do inventário florestal e em uma operacionalização mais eficaz.

Porém, para a construção adequada de modelos hipsométricos devem ser observados os diversos fatores que influenciam essa relação, tais como: posição sociológica, região, idade, densidade do plantio e práticas silviculturais em geral. Segundo Barros et al. (2002), vários modelos estatísticos podem adequar-se à relação hipsométrica. Batista et al. (2001) avaliaram o desempenho de modelos hipsométricos em três tipos de florestas e salientaram que os modelos respondem às particularidades da amostra utilizada no ajuste, e que a proporção de árvores em diferentes classes diamétricas influencia de forma diferenciada os diversos modelos ajustados, podendo super ou subestimar alturas.

Sabe-se que, para a correta aplicação de uma regressão, toda amplitude de variação dos dados deve ser medida. Portanto, a metodologia selecionada para escolher quais árvores da parcela que deverão ser medidas as alturas, terá influência no desempenho da relação hipsométrica. Nas metodologias tradicionais, a escolha das árvores a serem mensuradas geralmente se dá de maneira aleatória, como medir as cinco primeiras árvores da parcela ou as fileiras centrais, por exemplo. Ao adotar esse tipo de metodologia, não se garante a representatividade de toda amplitude dos diâmetros contidos na parcela. Pesonen et al. (2009) ao estudar Larix spp. mensurou altura das árvores de área basal média, as cinco maiores árvores e também a menor árvore baseado no diâmetro, dessa maneira garantindo a representatividade em toda amplitude diâmetro/altura das parcelas.

Dessa forma, neste trabalho, objetivou-se testar diferentes estratégias de ajustes hipsométricos, bem como avaliar modelos tradicionais e genéricos selecionados na literatura. Os ajustes foram feitos, utilizando três diferentes metodologias de coleta de dados em campo, ou seja, diferentes números de árvores utilizadas para proceder os ajustes. As estratégias adotadas foram realizadas a fim de obter informações concisas para a aplicação correta de relações hipsométricas na realização de inventários florestais e, consequentemente, diminuir custos e morosidade no processo de mensuração da variável altura.

\section{MATERIAL E MÉTODOS}

\subsection{Localização e caracterização da área de estudo}

Os dados utilizados para desenvolver o estudo foram obtidos em plantios de Eucalyptus sp., pertencentes a empresa Veracel Celulose S.A, situados na região sul do estado da Bahia, no município de Eunápolis. Segundo a classificação de Köppen a região apresenta como características básicas: temperaturas médias mensais superiores a $18^{\circ} \mathrm{C}$ e todos os meses com índices pluviométricos superiores a $60 \mathrm{~mm}$. Insere-se em faixa sazonal de baixa latitude, conferindo-lhe clima tropical, quente e úmido com cobertura vegetal de floresta (ASSIS et al., 2003).

As regiões provenientes da base de dados utilizada possuem diferentes características de produtividade, sendo a região 1 a de maior produtividade, a região 2 de produtividade intermediária e a região 3 de produtividade inferior, classificadas conforme fatores edáficos, climáticos e fisiográficos de cada local.

\subsection{Coleta de dados}

Para realização do trabalho, foram obtidos diâmetros e alturas reais de 782 árvores distribuídas em 36 parcelas, onde a altura de todas as árvores de cada parcela foi medida utilizando um hipsômetro Suunto. Cada parcela de $264 \mathrm{~m}^{2}$ contém 22 covas de plantio (resultante de um espaçamento de $4 \times 3 \mathrm{~m}$ ) distribuídas em 9 talhões, representando as idades de 3,5 e 7 anos, em cada região conforme produtividade (1, 2 e 3$)$.

As alturas dominantes das parcelas foram determinadas por meio do conceito de Assmann (PRODAN et al., 1997), o qual corresponde à altura média aritmética das 100 árvores de maior diâmetro por hectare.

\subsection{Modelos avaliados}

Foi avaliado o desempenho de três modelos tradicionais selecionados na literatura florestal e dois modelos genéricos. Os modelos foram ajustados, utilizando o programa de regressão linear Statgraphics Plus (1995). A estrutura matemática dos modelos utilizados se encontram na Tabela 1.

Cerne, Lavras, v. 16, n. 1, p. 22-31, jan./mar. 2010 
Tabela 1 - Estrutura matemática dos modelos hipsométricos avaliados.

Table 1 - Mathematic structure of hypsometric models evaluated.

\begin{tabular}{lcc}
\hline Nome & Modelo matemático & Fonte \\
\hline Prodan & $H-1,3=\frac{D A P^{2}}{\beta_{0}+\beta_{1} D A P+\beta_{2} D A P^{2}}+\varepsilon_{i}$ & $\begin{array}{c}\text { Prodan } \\
(1968)\end{array}$ \\
\hline Curtis & $\operatorname{Ln}(H)=\beta_{0}+\beta_{1} \frac{1}{D A P}+\varepsilon_{i}$ & $\begin{array}{c}\text { Curtis } \\
(1967)\end{array}$ \\
\hline $\begin{array}{l}\text { Parabólico } \\
\text { Scolforo }\end{array}$ & $H=\beta_{0}+\beta_{1} D A P+\beta_{2} D A P^{2}+\varepsilon_{i}$ & $\begin{array}{c}\text { Trorey } \\
(1932)\end{array}$ \\
\hline $\begin{array}{l}\text { Campos \& Leite } \\
\text { (2006) } \\
\text { modificado }\end{array}$ & $H=\exp \left[\beta+\frac{\beta_{1}}{D A P}+\operatorname{Ln}\left(H_{d o m}\right)\right]+\varepsilon_{i}$ & $\begin{array}{c}\text { Scolforo } \\
(1998)\end{array}$ \\
\hline
\end{tabular}

Sendo: $\mathrm{H}=$ altura total da árvore $(\mathrm{m}) ; \mathrm{H}_{\mathrm{dom}}=$ altura das árvores dominantes $(\mathrm{m}) ; \mathrm{DAP}=$ diâmetro à $1,30 \mathrm{~m}$ do solo $(\mathrm{cm})$; $\mathrm{Dg}=$ diâmetro médio quadrático $(\mathrm{cm}) ; \mathrm{I}=$ idade (anos); $\mathrm{Ln}=$ logarítmo neperiano; $\beta \mathrm{i}$ = parâmetros dos modelos a serem estimados; $\varepsilon_{\mathrm{i}}=$ erro da estimativa.

\subsection{Estratégia e metodologia de ajuste}

Os ajustes foram realizados simulando três diferentes metodologias de coleta de dados em campo, onde a primeira consistiu na utilização das 5 primeiras árvores medidas na parcela para a realização do ajuste, a segunda, feita com as 5 primeiras árvores medidas mais a adição de árvores dominantes, totalizando no máximo sete árvores por parcela. E, por último, com as 5 primeiras árvores juntamente com as árvores dominantes mais a adição de árvores que não foram representadas em todos os quartis baseados em diâmetro, avaliado por meio do Box Plot, onde o número de árvores adicionadas variou conforme representatividade da distribuição diamétrica na curva normal.

O Box Plot, também chamado de Diagrama dos cinco números, foi utilizado com intuito de verificar se havia árvores mensuradas em altura ao longo da dispersão da curva normal diamétrica. A construção do Box Plot seguiu a teoria descrita em diversos livros de estatística, tal como Bussab \& Morettin (2004).

O número de árvores utilizadas no ajuste dos modelos variou conforme a estratégia de ajuste adotada, bem como na forma que se construiu o ajuste, não ultrapassando 8 árvores por parcela. No caso da estratégia de ajuste por idade, uniu-se as árvores de todas as parcelas com a mesma idade. $\mathrm{O}$ mesmo aconteceu para a formação da base de dados para ajuste por região e para a combinação de idade e região (estrato).

As diferentes estratégias adotadas nas três diferentes metodologias de ajuste formaram 14 tratamentos, conforme apresentado na Tabela 2.

Tabela 2 - Tratamentos empregados no estudo.

Table 2-Treatments used in the study.

\begin{tabular}{cc}
\hline Tratamento & Estratégia \\
\hline 1 & Prodan (1968) por parcela \\
3 & Parabólico por parcela \\
4 & Curtis (1967) por parcela \\
5 & Prodan (1968) por idade \\
6 & Parabólico por idade \\
7 & Curtis (1967) por idade \\
8 & Prodan (1968) por região \\
9 & Parabólico por região \\
10 & Curtis (1967) por região \\
11 & Prodan (1968) por estrato \\
12 & Parabólico por estrato \\
13 & Curtis (1967) por estrato \\
14 & Scolforo (2005) (genérico) \\
\hline
\end{tabular}

Cerne, Lavras, v. 16, n. 1, p. 22-31, jan./mar. 2010 
Nos tratamentos 1, 2 e 3 ajustou-se um modelo para cada uma das 36 parcelas presentes, utilizando 5 árvores por parcela, totalizando 180 árvores; esses tratamentos também foram ajustados adicionando, no máximo, 2 árvores que possuíam altura dominante mensurada; e ajustados adicionando árvores selecionadas por meio do Box Plot, nos quartis não representados. Os tratamentos 4,5 e 6 foram ajustados com as idades de 3,5 e 7 anos, contendo cada idade 12 parcelas. $\mathrm{O}$ mesmo valor foi adotado para o ajuste por região. No caso dos tratamentos feitos por estrato, utilizouse a junção de idade e região para efetuar o ajuste dos modelos avaliados, contendo cada estrato 4 parcelas. Para avaliação dos modelos Scolforo (2005) e Campos \& Leite (2006), a base de dados foi completa, contendo valores de 180, 236 e 250 árvores, conforme metodologia de ajuste adotada.

\subsection{Seleção da melhor estratégia de ajuste}

A seleção da melhor estratégia de ajuste foi baseada nos seguintes parâmetros estatísticos: coeficiente de determinação ajustado $\left(\mathrm{R}^{2}\right)$ em porcentagem, erro padrão residual (Syx) em metros, análise gráfica de resíduos, análise de variância e teste de comparações múltiplas de Scott \& Knott (1974), ambos realizados por meio do software SISVAR desenvolvido por Ferreira (1999). O teste de comparações múltiplas de Scott-Knott foi escolhido por ser considerado robusto e por não apresentar resultados ambíguos, de acordo com Borges \& Ferreira (2003). Os valores do erro padrão residual foram transformados em metros para permitir a comparação entre modelos logaritimazados e não logaritimazados. Os valores do coeficiente de determinação foram ajustados para permitir a comparação entre modelos com diferente numero de parâmetros ajustados, conforme apresentado em Triola (1999).

Para seleção adequada da melhor estratégia de ajuste, foi realizada uma análise de variância, em delineamento inteiramente casualizado (DIC), onde os 14 tratamentos foram empregados, cada um contendo 782 repetições (sendo cada árvore uma unidade amostral), essas consistindo no valor do erro absoluto em metros de cada árvore presente na base total dos dados, fazendo, dessa forma, uma validação preditiva. Sendo então os valores mais próximos de zero ideais, visto menor variação da altura estimada em relação à altura real da árvore. Havendo interação significativa, fundamentou-se no teste de Scott $\&$ Knott (1974), gerado com os 782 valores de erro absoluto em metros de todos os dados por tratamento, para seleção da melhor estratégia de ajuste.

\section{RESULTADOS E DISCUSSÃO}

Os valores médios das estatísticas de precisão para seleção da melhor estratégia de ajuste e da melhor metodologia adotada são apresentados na Tabela 3.

As estratégias por parcela, por estrato e modelos genéricos, apresentaram os melhores valores quando observadas as estatísticas de precisão em todas as metodologias empregadas no estudo. Os valores de $\mathrm{R}^{2}$ foram altos para modelos genéricos, mostrando o potencial destes para estimativa da altura, conforme constatado por Leite \& Andrade (2003). Avaliando o Syx, para a metodologia utilizando as 5 primeiras árvores, o modelo de Curtis apresentou os melhores resultados em todas as estratégias de ajuste utilizadas. Nas demais metodologias, os ajustes feitos por parcela, estrato e modelos genéricos obtiveram as melhores estatísticas.

Avaliando o $\mathrm{R}^{2}$, o uso de árvores representando os quartis da distribuição diamétrica, melhorou as estatísticas de ajuste por parcela, quando comparadas às demais metodologias. Isso mostra que, se a opção de ajuste for por parcela, a representatividade em toda amplitude diâmetro/altura deve ser observada. Porém, ao avaliarmos conjuntamente os valores de Syx, verifica-se que os valores aumentam conforme são inseridas mais árvores para realização do ajuste. Esse fato ocorre em razão do aumento na amplitude de variação dos dados e, consequentemente, dos valores de Syx.

Por outro lado, de maneira geral, as estatísticas dos modelos pioraram nas estratégias de ajuste por região, idade e estrato. Esses resultados sinalizam para o potencial de ajuste por parcela, como já vem sendo utilizado em escala operacional nas empresas florestais. A opção de ajuste feita por estrato deve priorizar uma base de dados mais ampla e, preferencialmente, ajustando modelos que utilizam covariáveis, tais como modelos genéricos.

A inclusão de árvores representativas em todos os quartis propiciou uma melhora significativa nos ajustes. Essa metodologia não possibilitou a ocorrência da degeneração da relação hipsométrica (valores nulos do coeficiente de determinação), quando a estratégia de ajuste adotada foi por parcela, contrariando as demais formas de ajuste onde ocorreu a degeneração em algumas parcelas.

Para avaliação da qualidade dos ajustes, além do cálculo do erro padrão residual e do coeficiente de determinação, utilizou-se também a análise gráfica de resíduos. Foram verificadas diferenças entres os tratamentos, quando realizada a análise visual de gráficos.

Cerne, Lavras, v. 16, n. 1, p. 22-31, jan./mar. 2010 
Tabela 3 - Valores médios das estatísticas de precisão dos diferentes tratamentos e metodologias utilizados no estudo.

Table 3 - Mean values of the precision statistics for the treatments and methodologies used in the study.

\begin{tabular}{|c|c|c|c|c|c|c|c|}
\hline \multirow{3}{*}{ Tratamento } & \multirow{3}{*}{ Descrição } & \multicolumn{6}{|c|}{ Metodologias } \\
\hline & & \multicolumn{2}{|c|}{5 primeiras árvores } & \multicolumn{2}{|c|}{$\begin{array}{c}5 \text { primeiras + árvores } \\
\text { dominantes }\end{array}$} & \multicolumn{2}{|c|}{$\begin{array}{c}5 \text { primeiras + árvores em } \\
\text { todos os quartis }\end{array}$} \\
\hline & & Syx (m) & $\mathrm{R}^{2}(\%)$ & Syx (m) & $\mathrm{R}^{2}(\%)$ & Syx (m) & $\mathrm{R}^{2}(\%)$ \\
\hline 1 & Prodan (1968) por parcela & 0,5189 & 77,1962 & 0,5166 & 82,4026 & 0,5461 & 84,6904 \\
\hline 2 & Parabólico por parcela & 0,4769 & 79,6803 & 0,5087 & 82,9038 & 0,5409 & 85,1184 \\
\hline 3 & Curtis (1967) por parcela & 0,5603 & 84,2607 & 0,6031 & 84,0877 & 0,6304 & 86,2964 \\
\hline 4 & Prodan (1968) por idade & 1,3157 & 78,3437 & 1,4343 & 74,8664 & 1,4298 & 76,3718 \\
\hline 5 & Parabólico por idade & 1,3137 & 78,4072 & 1,4331 & 74,8924 & 1,4283 & 76,4097 \\
\hline 6 & Curtis (1967) por idade & 1,4342 & 76,2731 & 1,5395 & 73,5161 & 1,5485 & 74,9399 \\
\hline 7 & Prodan (1968) por região & 2,2924 & 81,6741 & 3,2556 & 61,9125 & 2,5441 & 79,1882 \\
\hline 8 & Parabólico por região & 2,3453 & 81,0602 & 2,5159 & 79,8486 & 2,5529 & 79,0759 \\
\hline 9 & Curtis (1967) por região & 2,5062 & 74,5028 & 2,6879 & 73,2559 & 2,7871 & 70,9267 \\
\hline 10 & Prodan (1968) por estrato & 0,9090 & 77,3741 & 0,8520 & 75,1414 & 0,9282 & 71,9839 \\
\hline 11 & Parabólico por estrato & 0,9066 & 77,4588 & 0,8488 & 75,3890 & 0,8118 & 79,8960 \\
\hline 12 & Curtis (1967) por estrato & 0,8954 & 78,3530 & 0,8524 & 77,6055 & 0,8723 & 80,6106 \\
\hline 13 & Scolforo (2005) & 1,0847 & 97,6853 & 0,8719 & 97,6931 & 0,8899 & 97,6931 \\
\hline 14 & Campos \& Leite (2006) & 1,0908 & 97,6440 & 0,8771 & 97,6958 & 0,8879 & 97,6405 \\
\hline
\end{tabular}

De um modo geral, os gráficos não apresentaram grandes tendenciosidades para quase todos os tratamentos adotados. Nos tratamentos 7, 8 e 9, nos quais os ajustes foram realizados por região, ocorreu uma subestimativa da altura quando analisados os menores valores de diâmetro, e para os maiores valores houve uma ligeira superestimação, fato este que ocorreu em todas as metodologias empregadas. Na Figura 1, mostram-se os gráficos de resíduo para os 14 tratamentos para a metodologia das primeiras 5 árvores. É importante ressaltar que os gráficos da Figura 1 foram elaborados com os resíduos de todas as árvores da base de dados, e não apenas com os dados de ajuste. Em razão da semelhança dos gráficos de resíduos obtidos da metodologia das 5 primeiras árvores com as outras metodologias de ajuste empregadas, os posteriores não serão apresentados.

A seleção decisória do modelo empregado mais eficaz, bem como da melhor estratégia de ajuste e da metodologia de coleta de dados adequadas, foi tomada com base na ANOVA. Os valores das análises de variância são apresentados nas Tabelas 4, 6 e 8 para as 3 diferentes metodologias.

Pela Tabela 4 foi detectado que houve diferença entre os tratamentos, implicando que pelo menos um tratamento diferiu dos demais. Para separação dos tratamentos foi realizado o teste de média de Scott-Knott. Os resultados são apresentados na Tabela 5. Para esta forma de ajuste, as melhores estratégias adotadas foram a de Curtis por parcela e os modelos genéricos de Scolforo (2005) e Campos \& Leite (2006). A pior estratégia de ajuste foi por região, confirmando a questão de super ou subestimativa observada nos gráficos de resíduos, apresentados na Figura 1.

Quando foram incluidas as árvores dominantes, de acordo com a Tabela 6 , foi detectada diferença entre os tratamentos e foi realizado um teste de média para separação dos mesmos (Tabela 7). Nesta metodologia, a diferença entre os tratamentos foi inferior à metodologia anterior, utilizando apenas as 5 primeiras árvores. Novamente a estratégia de Curtis por parcela e os modelos genéricos apresentaram os melhores resultados, assim como a estratégia por região teve o pior desempenho.

No caso da inserção das árvores representativas em todos os quartis, de acordo com a Tabela 8 , o valor de Fc foi altamente significativo. Na Tabela 9, mostra-se o teste de média, o qual evidenciou novamente o potencial da estratégia por parcela para o modelo de Curtis, seguido dos modelos genéricos. Novamente a estratégia por região teve pior desempenho. 
Estratégias e metodologias de ajuste de modelos hipsométricos...
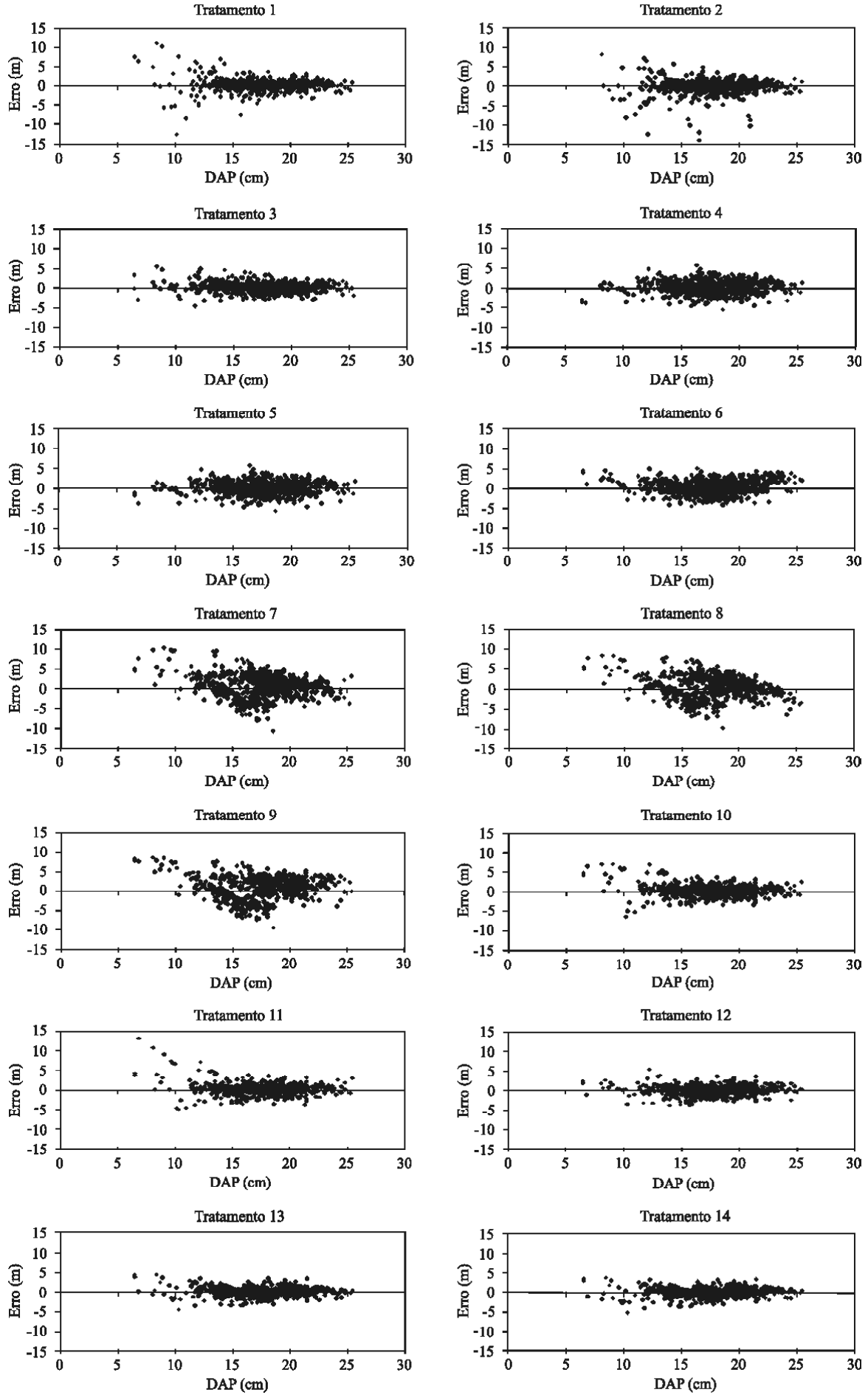

Figura 1 - Gráficos de resíduos obtidos com o uso das 5 primeiras árvores por parcela.

Figure 1-Residual plots obtained from the first five trees per plot.

Cerne, Lavras, v. 16, n. 1, p. 22-31, jan./mar. 2010 
Tabela 4 - Análise de variância para diferença entre altura real e estimada com o uso das 5 primeiras árvores por parcela.

Table 4-Analysis of variance for the difference between the observed and the estimated height values using the first five trees per plot.

\begin{tabular}{lccccc}
\hline FV & GL & SQ & QM & Fc & Pr $>$ Fc \\
\hline Tratamentos & 13 & $3.086,6430$ & 237,4341 & 88,237 & 0,000 \\
Erro & 10934 & $29.409,9974$ & 2,6898 & & \\
\hline Total corrigido & 10947 & $32.496,6404$ & & & \\
\hline
\end{tabular}

Tabela 5 - Teste de média Scott-Knott a 95\% de probabilidade dos valores médios de erro absoluto, para a base de dados das cinco primeiras árvores por parcela*.

Table 5 - Scott Knott means test at 95\% probability using the mean absolute error values, for the first five trees per plot database.

\begin{tabular}{|c|c|c|c|}
\hline Tratamento & Descrição & Média & \\
\hline 3 & Curtis (1967) por parcela & 0,727621 & $\mathrm{a}$ \\
\hline 13 & Campos \& Leite (2006) & 0,750639 & $\mathrm{a}$ \\
\hline 14 & Scolforo (2005) & 0,753197 & $\mathrm{a}$ \\
\hline 12 & Curtis (1967) por estrato & 0,854220 & $\mathrm{~b}$ \\
\hline 1 & Prodan (1968) por parcela & 0,934783 & $\mathrm{~b}$ \\
\hline 10 & Prodan (1967) por estrato & 0,951407 & $\mathrm{~b}$ \\
\hline 11 & Parabólico por estrato & 0,974425 & $\mathrm{~b}$ \\
\hline 5 & Parabólico por idade & 1,245524 & $\mathrm{c}$ \\
\hline 4 & Prodan (1968) por idade & 1,255754 & $\mathrm{c}$ \\
\hline 6 & Curtis (1967) por idade & 1,381074 & $\mathrm{~d}$ \\
\hline 2 & Parabólico por parcela & 1,428389 & $\mathrm{~d}$ \\
\hline 8 & Parabólico por região & 2,120205 & $\mathrm{e}$ \\
\hline 7 & Prodan (1968) por região & 2,121483 & $\mathrm{e}$ \\
\hline 9 & Curtis (1967) por região & 2,345269 & $\mathrm{f}$ \\
\hline
\end{tabular}

* As médias seguidas de letras iguais, não apresentam diferenças estatísticas na probabilidade de 95\% de acerto.

Tabela 6 - Análise de variância para diferença entre altura real e estimada com inclusão de árvores dominantes.

Table 6-Analysis of variance for the difference between the observed and the estimated height values with the inclusion of the dominant trees.

\begin{tabular}{lccccc}
\hline FV & GL & SQ & QM & Fc & Pr $>$ Fc \\
\hline Tratamentos & 13 & $5.014,8199$ & 385,7554 & 6,699 & 0,000 \\
Erro & 10934 & $629.631,3442$ & 57,5847 & & \\
\hline Total corrigido & 10947 & $634.646,1641$ & & & \\
\hline
\end{tabular}

Cerne, Lavras, v. 16, n. 1, p. 22-31, jan./mar. 2010 
Tabela 7 - Teste de média Scott-Knott a 95\% de probabilidade dos valores médios de erro absoluto, para a base de dados com inclusão das alturas dominantes*.

Table 7 - Scott-Knott means test at 95\% probability using the mean absolute error values, for the database with inclusion of the dominant trees.

\begin{tabular}{|c|c|c|c|}
\hline Tratamento & Descrição & Média & \\
\hline 3 & Curtis (1967) por parcela & 0,730035 & $\mathrm{a}$ \\
\hline 14 & Scolforo (2005) & 0,798608 & $\mathrm{a}$ \\
\hline 13 & Campos \& Leite (2006) & 0,811907 & $\mathrm{a}$ \\
\hline 12 & Curtis (1967) por estrato & 0,880390 & $\mathrm{a}$ \\
\hline 11 & Parabólico por estrato & 0,913198 & $\mathrm{a}$ \\
\hline 10 & Prodan (1968) por estrato & 0,988536 & $\mathrm{a}$ \\
\hline 5 & Parabólico por idade & 1,271866 & $\mathrm{a}$ \\
\hline 4 & Prodan (1968) por idade & 1,274585 & $\mathrm{a}$ \\
\hline 2 & Parabólico por parcela & 1,327193 & $\mathrm{a}$ \\
\hline 6 & Curtis (1967) por idade & 1,375852 & $\mathrm{a}$ \\
\hline 8 & Parabólico por região & 2,150214 & $\mathrm{~b}$ \\
\hline 1 & Prodan (1968) por parcela & 2,192029 & $\mathrm{~b}$ \\
\hline 9 & Curtis (1967) por região & 2,356858 & $\mathrm{~b}$ \\
\hline 7 & Prodan (1968) por região & 2,965258 & $\mathrm{~b}$ \\
\hline
\end{tabular}

* As médias seguidas de letras iguais, não apresentam diferenças estatísticas na probabilidade de $95 \%$ de acerto.

Tabela 8 - Análise de variância para diferença entre altura real e estimada com inclusão de árvores representativas em todos os quartis.

Table 8-Analysis of variance for the difference between the observed and the estimated height values with inclusion of the trees representative in all the quartiles.

\begin{tabular}{lccccc}
\hline FV & GL & SQ & QM & Fc & Pr>Fc \\
\hline Tratamentos & 13 & $3.253,6424$ & 250,2802 & 124,3400 & 0,000 \\
Erro & 10934 & $22.008,7379$ & 2,0129 & & \\
\hline Total corrigido & 10947 & $25.262,3803$ & & & \\
\hline
\end{tabular}

Portanto, para resultado geral, visto a estratégia de Curtis por parcela ter liderado todos os testes de médias realizados, foi feita uma nova análise de variância contendo apenas esta estratégia de ajuste, nas três diferentes metodologias adotadas. A seguir estão listados os resultados.

$\mathrm{O}$ valor de Fc da Tabela 10 indica que houve diferença entre os tratamentos, fazendo-se um teste de média para separação dos mesmos (Tabela 11).
Analisando o teste de média, verifica-se que apenas a metodologia utilizando as 5 primeiras árvores se difere das demais. Portanto, a inclusão de mais árvores no ajuste da relação hipsométrica por parcela propicia melhores resultados na estimativa da altura, e ainda a inclusão de árvores representativas em todas as classes de variabilidade de diâmetro implicou em um menor valor médio do erro absoluto. 
Tabela 9 - Teste de média Scott-Knott a 95\% de probabilidade dos valores médios de erro absoluto, para a base de dados com inclusão de árvores representativas em todos os quartis*.

Table 9 - Scott-Knott means test at 95\% probability using the mean absolute error values, for the database with inclusion of the trees representative in all the quartiles.

\begin{tabular}{|c|c|c|c|}
\hline Tratamento & Descrição & Média & \\
\hline 3 & Curtis (1967) por parcela & 0,622762 & a \\
\hline 14 & Scolforo (2005) & 0,749361 & $\mathrm{~b}$ \\
\hline 13 & Campos \& Leite (2006) & 0,777494 & $\mathrm{~b}$ \\
\hline 1 & Prodan (1968) por parcela & 0,778772 & $\mathrm{~b}$ \\
\hline 12 & Curtis (1967) por estrato & 0,824808 & $\mathrm{~b}$ \\
\hline 2 & Parabólico por parcela & 1,069054 & $\mathrm{c}$ \\
\hline 5 & Parabólico por idade & 1,221228 & $\mathrm{~d}$ \\
\hline 4 & Prodan (1967) por idade & 1,228900 & $\mathrm{~d}$ \\
\hline 11 & Parabólico por estrato & 1,283887 & $\mathrm{e}$ \\
\hline 6 & Curtis (1968) por idade & 1,363171 & $\mathrm{e}$ \\
\hline 10 & Prodan (1968) por estrato & 1,411765 & $\mathrm{e}$ \\
\hline 7 & Prodan (1968) por região & 2,109974 & $\mathrm{f}$ \\
\hline 8 & Parabólico por região & 2,135550 & $\mathrm{f}$ \\
\hline 9 & Curtis (1967) por região & 2,393862 & $\mathrm{~g}$ \\
\hline
\end{tabular}

* As médias seguidas de letras iguais, não apresentam diferenças estatísticas na probabilidade de 95\% de acerto.

Tabela 10 - Análise de variância para diferença entre altura real e estimada no tratamento de Curtis por parcela, nas três diferentes metodologias de ajuste adotadas.

Table 10 - Analysis of variance for the difference between the observed and the estimated height values for the Curtis per plot treatment, considering the three different adjustment methods.

\begin{tabular}{lccccc}
\hline FV & GL & SQ & QM & Fc & Pr $>$ Fc \\
\hline Tratamentos & 2 & 4,4433 & 2,2217 & 3,1880 & 0,0414 \\
Erro & 2343 & $1.632,5358$ & 0,6968 & & \\
\hline Total corrigido & 2345 & $1.636,9791$ & & &
\end{tabular}

Tabela 11 - Teste de média Scott-Knott a 95\% de probabilidade dos valores médios de erro absoluto, no tratamento 1 pelas três diferentes metodologias de ajuste empregadas no estudo*.

Table 11 - Scott-Knott means test at 95\% probability using the mean absolute error values, for treatment 1 considering the three different adjustment methods.

\begin{tabular}{lcc}
\hline Descrição & Média & \\
\hline Curtis por parcela utilizando Box Plot & 0,622762 & $\mathrm{a}$ \\
Curtis por parcela utilizando alturas dominantes & 0,658568 & $\mathrm{a}$ \\
Curtis por parcela utilizando 5 árvores & 0,727621 & $\mathrm{~b}$ \\
\hline
\end{tabular}

\footnotetext{
* As médias seguidas de letras iguais, não apresentam diferenças estatísticas na probabilidade de $95 \%$ de acerto.
}

Cerne, Lavras, v. 16, n. 1, p. 22-31, jan./mar. 2010 


\section{CONCLUSÕES}

A inclusão de mais árvores no ajuste da relação hipsométrica por parcela propiciou melhorias na estimativa da variável altura. Em todas as formas de ajuste adotadas, a estratégia de Curtis por parcela apresentou menores valores médios de erro absoluto, conforme teste de média, e também as medidas de precisão utilizadas foram superiores às demais estratégias. Dessa forma, confirma-se a preferência de ajustes feitos por parcela, onde se tem uma menor área possibilitando o controle de fatores que interferem na relação hipsométrica, tais como espécie, idade, densidade de plantio e outros.

Os modelos genéricos também obtiveram resultados satisfatórios quando analisados as medidas de precisão, distribuição de gráficos e teste de média. Elucidando que o uso de modelos genéricos implica em um processo menos moroso na prática do inventário florestal, visto que, o ajuste de apenas uma equação poderá ser aplicada em toda a base de dados.

\section{REFERÊNCIAS BIBLIOGRÁFICAS}

ASSIS, D. S.; MANZATTO, C. V.; COUTINHO, S. C. Zoneamento agroecológico de microbacias hidrográficas da costa do descobrimento: município de Porto Seguro e Santa Cruz Cabrália, Bahia. Rio de Janeiro: Embrapa Solos, 2003.

BARROS, D. A.; MACHADO, S. A.; ACERBI JÚNIOR, F. W.; SCOLFORO, J. R. S. Comportamento de modelos hipsométricos tradicionais e genéricos para plantações de Pinus oocarpa em diferentes tratamentos. Boletim de Pesquisa Florestal, Colombo, n. 45, p. 3-28, 2002.

BATISTA, J. L. F.; COUTO, H. T. Z.; MARQUESINI, M. Desempenho de modelos de relações hipsométricas: estudo em três tipos de floresta. Revista Scientia Forestalis, Piracicaba, n. 60 , p. 149-163, 2001.

BORGES, L. C.; FERREIRA, D. F. Poder e taxas de erro tipo I dos testes Scott-Knott, Tukey e Student-Newman-Keuls sob distribuições normal e não normais dos resíduos. Revista de Matemática e Estatística, São Paulo, v. 21, n. 1, p. 67-83, 2003.

BUSSAB, W. O.; MORETTIN, P. A. Estatística básica. 5. ed. São Paulo: Saraiva, 2004. 526 p.

CAMPOS; LEITE. Mensuração florestal: perguntas e respostas. 2. ed. Viçosa, MG: UFV, 2006. 470 p.

CURTIS, R. Height-diameter and height-diameter-age equations for second-growth Douglas-fir. Forest science, Amsterdam, v. 13 , n. 4 , p. $365-375,1967$.
FERREIRA, D. F. SISVAR: sistema de análise de variância para dados balanceados. Versão 4.0. Lavras: DEX/UFLA, 1999. Software.

KER, J.; SMITH, J. Sampling for height-diameter relationships. Journal of Forestry, Amsterdam, v. 55, n. 3, p. 205-207, 1957.

LEITE, H. G.; ANDRADE, V. C. L. Importância das variáveis altura dominante e altura total em equações hipsométricas e volumétricas. Revista Árvore, Viçosa, v. 27, n. 3, p. 301-310, 2003.

PESONEN, A.; EERIKÄINEN, K.; MALTAMO, M.; TAHVANAINEN, T. Models for predicting tree and stand development on larch plantations in Hallormsstaður, Iceland. New Forests, Netherlands, v. 37, n. 1, p. 63-83, 2009.

PRODAN, M. Forest biometrics. Oxford: Pergamon, 1968. $447 \mathrm{p}$.

PRODAN, M. et al. Mensura forestal. San José: IICA, 1997. 586 p. (Serie Investigación y Educación en Desarrollo Sostenible).

SCOLFORO, J. R. S. Biometria florestal: parte I: modelos de regressão linear e não linear: parte II: modelos para relação hipsométrica, volume, afilamento e peso da matéria seca. Lavras: UFLA/FAEPE, 2005. 352 p.

SCOTT, A. J.; KNOTT, M. A cluster analysis method for grouping means in the analysis of variance. Biometrics, Washington, v. 30, p. 507-512, Sept. 1974.

SILVA, G. F.; XAVIER, A. C.; RODRIGUES, F. L.; PETERNELLI, L. A. Análise da influência de diferentes tamanhos e composições de amostras no ajuste de uma relação hipsométrica para Eucalyptus grandis. Revista Árvore, Viçosa, v. 31, n. 4, p. 685-694, 2007.

STATGRAPHICS PLUS. User manual. Rockville: Manugistics, 1995.

THIERSCH, C. R. Uma nova proposta metodológica para cubagem rigorosa e ajuste de modelos hipsométricos. 2007. 75 p. Tese (Doutorado em Engenharia Florestal) - Universidade Federal de Lavras, Lavras, 2007.

TRIOLA, M. F. Introdução à estatística. Rio de Janeiro: LTC, 1999. $410 \mathrm{p}$.

TROREY, L. G. A mathematical method for the construction of diameter height curves based on site. The Forestry Chronicle, Ottawa, v. 8, n. 2, p. 121-132, 1932.

Cerne, Lavras, v. 16, n. 1, p. 22-31, jan./mar. 2010 